A LETTERS JOURNAL EXPLORING THE FRONTIERS OF PHYSICS

\title{
OFFPRINT
}

\section{High nonlinear optical anisotropy of urea nanofibers}

D. Isakov, E. De Matos Gomes, M. Belsley, B. Almeida, A.

Martins, N. Neves and R. Reis

EPL, 91 (2010) 28007

Please visit the new website

www.epljournal.org 


\section{TARGET YOUR RESEARCH WITH EPL}

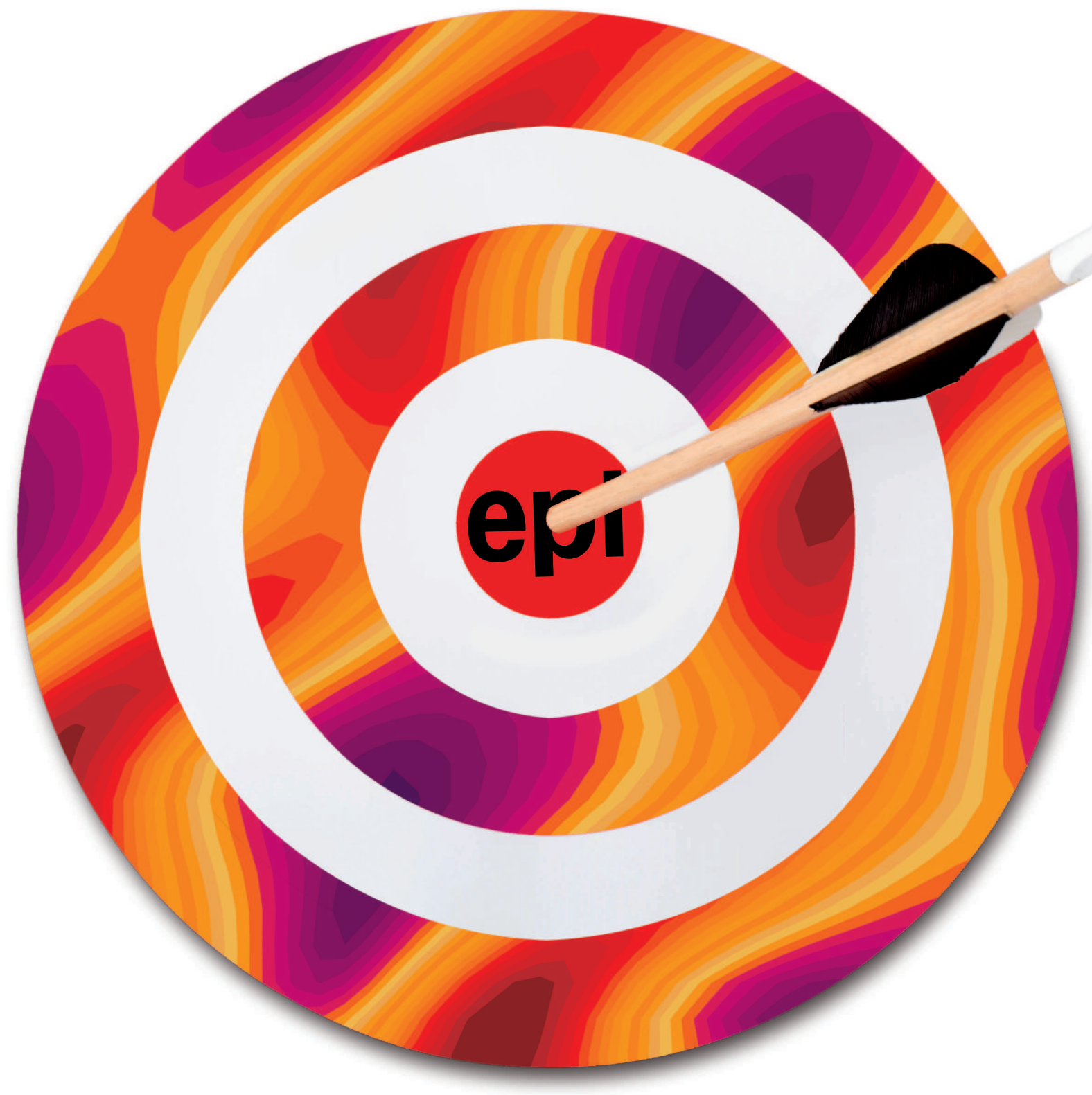

Sign up to receive the free EPL table of contents alert. www.epljournal.org/alerts 


\title{
High nonlinear optical anisotropy of urea nanofibers
}

\author{
D. Isakovi(a), E. De Matos Gomes ${ }^{1}$, M. Belsley ${ }^{1}$, B. Almeida ${ }^{1}$, A. Martins ${ }^{2}$, N. Neves $^{2}$ and R. Reis $^{2}$ \\ ${ }^{1}$ University of Minho, Center of Physics, Campus de Gualtar - 4710-057 Braga, Portugal, EU \\ 2 3B's Research Group - Biomaterials, Biodegradables and Biomimetics, Department of Polymer Engineering, \\ University of Minho - AvePark, Zona Industrial da Gandra, S. Cláudio do Barco, 4806-909 Caldas das Taipas, \\ Guimarães, Portugal, EU
}

received 30 March 2010; accepted in final form 18 July 2010 published online 5 August 2010

\author{
PACS 81.07.-b - Nanoscale materials and structures: fabrication and characterization \\ PACS 68.35.bm - Polymers, organics \\ PACS $42.79 . \mathrm{Nv}$ - Optical frequency converters
}

\begin{abstract}
Nanofibers consisting of the optically nonlinear organic molecule urea embedded in both poly(ethylene oxide) (PEO) and poly(vinyl alcohol) (PVA) polymers were produced by the electrospinning technique. The second-harmonic generation produced by aligned fiber mats of these materials displays a strong dependence on the polarization of the incident light. In PVA-urea nanofibers the effectiveness in generating of the second-harmonic light is as high as that of a pure urea powder with an average grain size of $110 \mu \mathrm{m}$. The results suggest that single crystalline urea nanofibers were achieved with a long-range crystalline order extending into the range of $2-4 \mu \mathrm{m}$ with PVA as the host polymer.
\end{abstract}

Copyright (C) EPLA, 2010

Over the past decade intensive efforts have been focused on the nanoscale engineering and design of materials with enhanced optical properties. Although it has long been recognized that organic nanoparticles with strong nonlinear optical responses can offer a broad range of potential applications ranging from fluorescence nanoprobes to frequency doubled nanosources [1], the production and characterization of nanoparticles exhibiting strong quadratic optical nonlinearities is a recent topic. Only a very few examples have been recently reported in the literature [2-5]. Nonetheless, the architectural flexibility, intrinsically good hyperpolarizability, low cost and unique size-dependent optoelectronic properties make organic nanostructures strong candidates for engineering devices based on their unique optical responses [6-8]. Indeed nanostructured optically nonlinear aligned assemblies have aroused great interest [9-11] and could find a wide range of applications ranging from environmental sensors to those based upon photonic crystal structures and light waveguides. Additionally, organic nanostructures can be easily integrated with conventional semiconductor devices, providing extra functionality to existing photonic circuits and components $[12,13]$.

Recent studies have revealed that organic molecules embedded within the fibers produced by electrospinning

(a) E-mail: dmitry@fisica.uminho.pt can possess a high degree of molecular orientation [14-16]. This characteristic can significantly expand the list of nanofiber applications due to the anisotropic properties of such objects. In particular, they can be used in the field of nonlinear optics; for example the second-harmonic generation ( $\mathrm{SHG}$ ) response of a material is largely dependent on the local symmetry and macroscopic orientation of the constituent molecule ensembles. In this regard organic nonlinear optical molecules are especially interesting. Since they can be readily embedded within a polymer fiber matrix, they have the potential to serve as the functional or multifunctional core. In order to realize this potential it is necessary to obtain molecular assemblies with the desired long-range orientational order over significant distances.

In this work we explore a possible application that makes use of the unique properties of the nanofiber assemblies with a strong nonlinear optical response. In-plane well-aligned nanofiber mats of two different polymer-urea complexes were produced by the electrospinning technique. Urea is one of the earliest organic nonlinear optical materials to have been developed commercially. It has been used to produce second-harmonic generation (SHG) in the UV and as the basis for optical parametric generation. Urea in the bulk crystalline form has a significant nonlinear optical response (its effective second-order nonlinear coefficient is roughly 2.5 times larger than that 


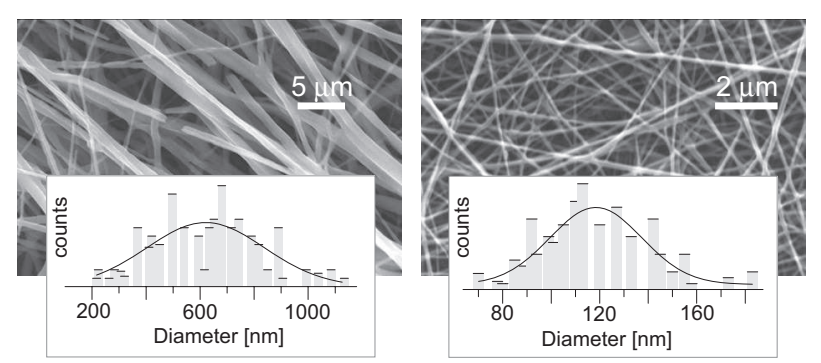

Fig. 1: SEM micrographs and thickness distribution of electrospun PEO-urea (left) and PVA-urea (right) nanofibers.

of ADP), a high degree of birefringence, and a relatively high laser damage threshold (about $3 \mathrm{GW} / \mathrm{cm}^{2}$ at $532 \mathrm{~nm}$ with $10 \mathrm{~ns}$ pulses). Our aim was to obtain low-dimensional sub-micrometer scaled nonlinear optical active materials based on this compound. Two different polymer matrices for the nanofibers were chosen, poly(ethylene oxide) (PEO) and poly(vinyl alcohol) (PVA). Both of the polymers are soluble in water (as urea is) and a 1:1 polymerto-urea mass ratio was used in both cases. However, as we show below, the SHG response of urea in these two polymer matrices is significantly different. PEO-urea fibers were prepared as described in ref. [15].

To produce the in-plane-aligned fiber mats, the precursor solutions were loaded into a syringe which was pumped at $1.0 \mathrm{ml} / \mathrm{h}$ flow rate with $10-12 \mathrm{kV}$ applied between the needle and the collector drum. The collector drum was kept at a distance of $12 \mathrm{~cm}$ and rotated at a rate of $100 \mathrm{rpm}$ (as described, for instance, in [17]). The fibers alignment was immediately verified by imaging the obtained fiber mat using Carl Zeiss Axio Imager.Z1m microscope coupled with an AxioCam MRm CCD camera and performing a two-dimensional Fourier transform. For accurate morphology evaluation the electrospun fibers have been randomly collected on collector plate and visualized by a Leica Cambridge S360 scanning electron microscope. Figure 1 presents the scanning electron micrographs and fiber diameter distribution of electrospun PEO- and PVA-urea random fibers mat. As can be seen, PEO-urea fiber diameter varies from $410 \mathrm{~nm}$ to $840 \mathrm{~nm}$, whereas the diameters of the obtained PVA-urea fibers are significantly smaller and more narrowly distributed between $100 \mathrm{~nm}$ and $140 \mathrm{~nm}$.

The X-ray diffraction measurements have been used to characterize the crystalline structure of the obtained electrospun PEO- and PVA-urea nanofiber mats. Figure 2 shows the diffractograms obtained using $2 \Theta$ incidence. As has been previously reported, in the electrospun PEO-urea nanofibers, the urea molecules combine with the polymer to form an inclusion complex having an orthorhombic unit cell [15]. The urea molecules are arranged as dimers forming channels along the fiber axis and the polymer chains are interlaced between these ribbon-like urea layers, also forming channels along the fiber axis. However, as can be seen from the X-ray diffraction patterns, the structure of PVA-urea nano fibers is quite different. There is no

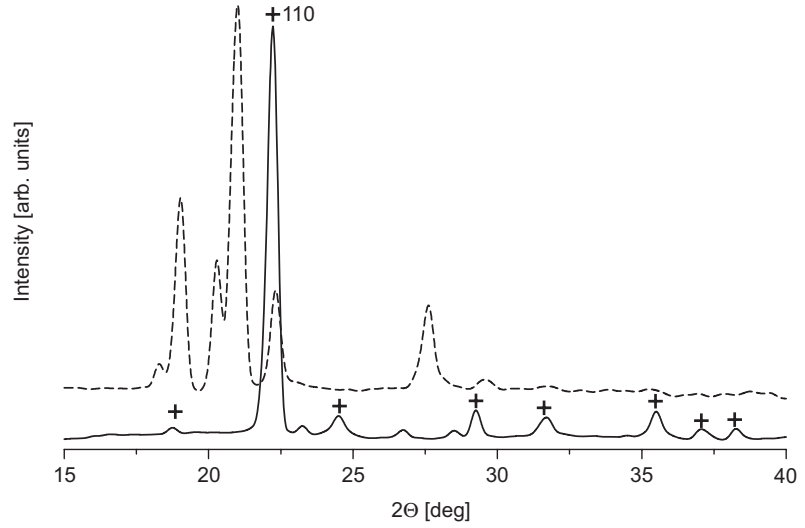

Fig. 2: X-ray diffraction patterns of electrospun fibers of PEOurea (dashed line) and PVA-urea (solid line). Crosses indicate diffraction peaks of pure crystalline urea.

evidence of the formation of an inclusion complex between the PVA polymer and urea molecules. Instead of inclusion complexes, the urea in the electrospun PVA-urea nano fibers form quasi-one-dimensional crystalline cores embedded within the PVA fibers, displaying the same tetragonal unit cell as seen in bulk pure urea crystals. What is more, the obtained urea crystalline cores display a surprisingly strong orientation - the urea lies predominantly in the (110) plane with polarizable axis parallel to the substrate.

Prior to the SHG measurements, the quantitative evaluation of the degree of alignment between fibers by a 2D Fourier transform (FT) image analysis technique with high-frequency-in-the-center representation of the transformed snapshot was carried out. The angular distribution of the fibers axes then has been plotted from an integral of a profile line rotated $180^{\circ}$ around the center of the 2D FT image [18]. A convenient measure of the degree of fiber orientation is the "orientational index" $(O I)$ which characterizes the fraction of parallel fibers along a given angle $\theta_{m}$,

$$
O I(\theta)=\left(2 \cdot\left\langle\cos ^{2} \theta_{m}\right\rangle-1\right) \cdot 100 \%,
$$

where

$$
\left\langle\cos ^{2} \theta_{m}\right\rangle=\frac{\int_{-90}^{90} I(\theta) \cos ^{2}\left(\theta-\theta_{m}\right) \mathrm{d} \theta}{\int_{-90}^{90} I(\theta) \mathrm{d} \theta} .
$$

Figure 3 shows an example of an initial photograph of an aligned in-plane array of PEO-urea nanofibers together with its 2D FT representation and angular power spectra density distribution. The orientational index, obtained from this sample is $O I \approx 70 \%$ which is evidence of the high degree of the average alignment over the nanofibers mat. The solid curve in fig. 3(c) represents the angular variation of the $O I$ for PVA-urea nanofibers. The $O I$ of PVA-urea fibers does not exceed $50 \%$. Assuming identical experimental conditions for the fabrication of the fibers from solution, the lower degree of alignment obtained 

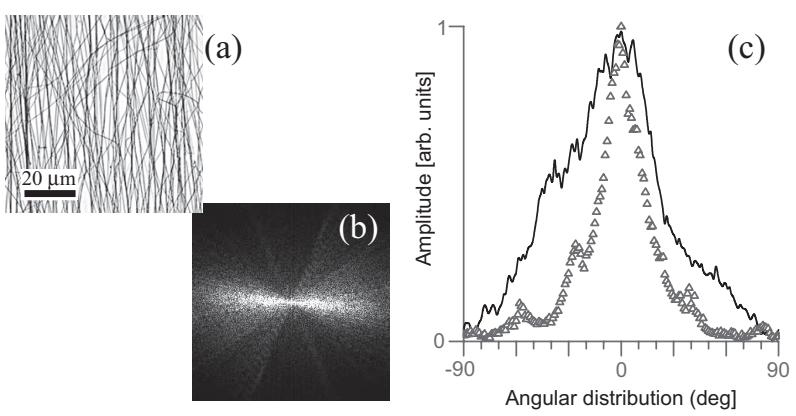

Fig. 3: Initial snapshot of electrospun PEO-urea nanofibers (a) and the high-frequency-in-the-center Fourier-transformed image (b). (c) Angular power spectra distribution of aligned PEO-urea fibers with an orientation index $O I=69.6 \%$; the solid curve corresponds to the PVA-urea nanofibers with $O I=48.6 \%$.

with PVA-urea nanofibers might be due to the different electrical conductivities of the initial solutions (in fact, PEO-urea precursor solution has a conductivity about 1.6 times higher than the PVA-urea solution). This difference could also be responsible for the different morphology of the electrospun fiber mats [19].

Monitoring the polarization dependence of SHG allows one both to determine the degree of alignment of the NLO active molecules with respect to the fiber axis [20] as well as to estimate the size of the single-domain crystalline regions over which a favorable alignment of the molecules is maintained within the crystals. To carry out such measurements, the linearly polarized fundamental beam at $1064 \mathrm{~nm}$ from a Nd:YAG laser was directed to be normally incident to the fiber mat. Appropriate color and interference filters were employed to discriminate against the scattered infrared fundamental light. The SHG light was detected with a polarization either parallel or perpendicular to the fiber array direction ( $q-p$ and $q-s$ configuration, respectively). The analyzed signal was recorded by a photomultiplier connected to a gated Boxcar integrator. To take into account the possibility of surface second-harmonic generation by the fibers, the SHG signal of pure PEO and pure PVA fiber mats was also measured. Since the signals from the pure polymer fibers were less than $0.1 \%$ of PEO-urea fiber mat, the surface SHG effect can be neglected. The two-photon fluorescence effect is assumed to be negligible due to the absence of any significant absorption by urea over the wavelength range extending from 200 to $1400 \mathrm{~nm}$ [21].

Figure 4 shows the polar variation of the observed second-harmonic signals as a function of the polarization direction of the fundamental $1064 \mathrm{~nm}$ beam, obtained in PEO-urea and PVA-urea aligned nanofiber arrays for a fixed analyzer orientation. The SHG polarization response in PEO-urea fibers is seen to be nearly independent of the analyzer orientation, implying a weak nonlinear optical active molecular orientation. Conversely, the polarization dependence of the SHG response of the PVA-urea aligned (a)

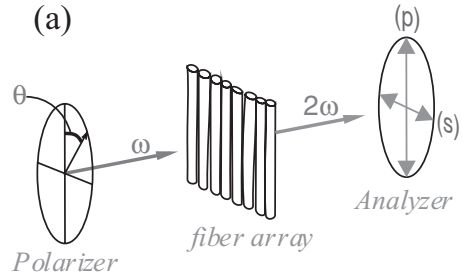

(b)

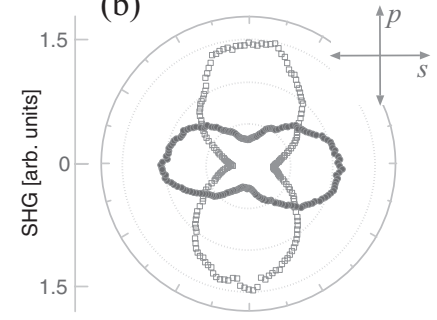

(c)

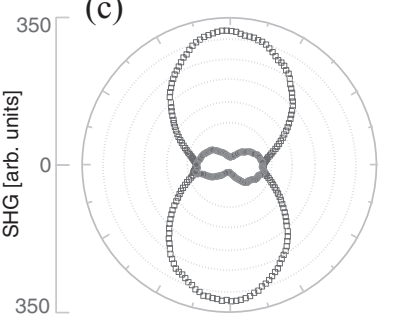

Fig. 4: (a) SHG ellipsometry setup sketch. (b), (c) SHG rotational responses of the polarization direction of $\mathrm{PEO}$-urea and PVA-urea nanofibers, respectively. The arbitrary relative intensity scale is the same in both graphs.

fibers array reveals a strong orientation of optically nonlinear active molecules along fibers. Taking into account that the twofold axis of urea is in the (110) plane, this strongly anisotropic SHG polarization response obtained from PVA-urea nano fibers array, implies that the urea dipole moment (which coincides with the tetragonal $c$-axis) is predominantly oriented along the fibers longitudinal axis. Note that the second-order susceptibility tensor of urea has only two independent coefficients (tetragonal point group $\overline{4} 2 m) d_{36}$ and $d_{14}$. For the $q-p$ configuration, with the detected second-harmonic electric field oriented along the fibers axis, the main contribution of the SHG intensity $I_{2 \omega}$ originates from $d_{36}: I_{2 \omega} \propto d_{36} \sin ^{4}(\theta)$. On the other hand, a perfectly aligned urea crystal with the $x$ and $y$ dielectric axes oriented at 45 degrees relative to the fiber mat plane, would result in a destructive cancellation of the $x$ and $y$ SHG components in the $q-s$ configuration. The slight nonzero SHG polarization response actually observed probably results from the less than perfect spatial fiber alignment. Scattering of the strong fundamental light by fibers in the topmost layer of the mat may also contribute.

Finally, to evaluate the nonlinear optical susceptibility of the obtained polymer shell-urea nanofibers, the SHG from urea crystal powder with $100-120 \mu \mathrm{m}$ grain size was measured as a reference sample. We have estimated the effective nonlinearity of the randomly distributed fibers through the expression

$$
d_{\mathrm{eff}}=d_{\mathrm{eff}}^{\mathrm{pwd}} \cdot \sqrt{\frac{I_{2 \omega} l^{\mathrm{pwd}}}{I_{2 \omega}^{\mathrm{pwd}} l}},
$$

where $l^{\text {pwd }}$ is cell length of the powder sample and $l$ is the thickness of the fiber mesh. Randomly oriented fiber mats, such as those imaged in fig. 1, were used to mimic 
the random orientation of crystals in the powder sample. Using the above relation we found that in randomly aligned fiber meshes $d_{\mathrm{eff}}^{\mathrm{PVA}-\text { urea }}=d_{\mathrm{eff}}^{\text {urea }} \times 1.061 \pm 0.122$, when $d_{\mathrm{eff}}^{\mathrm{PEO}-\text { urea }}=d_{\mathrm{eff}}^{\text {urea }} \times 0.019 \pm 0.042$. The strong difference in the SHG responses PVA-urea and PEO-urea fibers has structural nature. In other words, we believe that in PVA-urea nano fibers the SHG response is from urea nanocrystals which are embedded into the polymer matrix, while in PEO-urea the frequency doubling response is that of an urea inclusion complex. The fact that the nonlinear susceptibility of the PVA-urea nanofibers is similar to the susceptibility of the bulk crystalline urea (the SHG from the PVA-urea fiber scaffold is extremely bright), implies that the crystals embedded in the PVA fiber matrix are functional urea crystals with a length that is comparable to the grain size above which the plateau is reached for urea in the Kurtz powder test, namely in the range of $2-4 \mu \mathrm{m}$ [21]. As a result, we conclude that the PVA-urea nanofibers consist of a polymer fiber matrix with embedded aligned chains of urea cylinder-like crystals with typical size on the order of $100 \mathrm{~nm}$ in thickness and around $3 \mu \mathrm{m}$ in length.

The results show that polymer nanofibers embedded with optically nonlinear urea give rise to a strong anisotropic response of the second-harmonic generation as a function of the polarization direction of the incident light. The macroscopic orientation of small nonlinear active molecules can improve the optical properties of the materials, drastically exceeding those of the randomly oriented. The results obtained suggest that the choice of the polymer host is important and suggests that the formation of inclusion complexes, as occurred with the PEO fibers should be avoided. We speculate that the preferential crystal alignment along the fiber axis that we have obtained for PVA-urea fibers will be attainable for the majority of organic molecules able to form hydrogen bonds. Producing nanofibers with oriented crystallization of included organic complexes possessing high nonlinear optical efficiencies represents a significant step toward the realization of a host of various practical applications. For example these nanofibers could be used to provide a structured quasi-one-dimensional source of visible light by conversion of spatially uniform infrared light. Nanofibers formed with chromophores endowed with even high nonlinear molecular polarizabilities could serve as a promising organic electro-optical device prototypes, with numerous potential applications ranging from a variable intensity source of second-harmonic light to the creation of locally patterned light sources with variations in the spatial profile, polarization or even optical phase that could be employed in a variety of microscopy or lab-on-a-chip tasks.
This work was carried out in the frame of CIÊNCIA-2007 program (reference UMINHO-CF06) and financially supported by Fundação para a Ciência e Tecnologia, PTDC/CTM/105597/2008.

\section{REFERENCES}

[1] Nakayama Y., Pauzauskie P. J., Radenovic A., Onorato R. M., Saykally R. J., Liphardt J. and YANG P., Nature, 447 (2007) 1098.

[2] Brewer J., Schiek M., Wallmann I. and Rubahn H.-G., Opt. Commun., 281 (2008) 3892.

[3] Brewer J., Schiek M., Lutzen A., Al-Shamery K. and Rubahn H.-G., Nano Lett., 6 (2006) 2656.

[4] Humbert C., Busson B., Abid J., Six C., Girault H. and TAdjEDdine A., Electrochim. Acta, 50 (2005) 3101.

[5] Yi T., Clement R., Haut C., Catala L., Gacoin T., Tancrez N., Ledoux I. and Zyss J., Adv. Mater., 17 (2005) 335.

[6] Spano F. and Mukamel S., Phys. Rev. A, 40 (1989) 5783.

[7] Firestone K., Reid P., Lawson R., Jang S.-H. and Daltonet L. R., Inorg. Chem. Acta, 357 (2004) 3957.

[8] Duboisset J., Russier-Antoine I., Benichou E., Bachelier G., Jonin C. and Brevet P. F., J. Phys. Chem. C, 113 (2009) 13477.

[9] Schneider G. J. and Watson G. H., Appl. Phys. Lett., 83 (2003) 5350.

[10] Jagannathan R., Irvin G., Blanton T. and Jagannathan S., Adv. Funct. Mater., 16 (2006) 747.

[11] Zhang H. F., Hussain T., Brust M., Butler M. F., Rannard S. P. and Cooper A. I., Nat. Mater., 4 (2005) 787.

[12] Schiek M., Balzer F., Al-Shamery K., Brewer J. R., Lotzen A. and Rubahn H.-G., Small, 4 (2008) 176.

[13] Benight S., Bale D., Olbricht B. and Dalton L. R., J. Mater. Chem., 19 (2009) 7466.

[14] Kongkhlang T., Tashiro K., Kotaki M. and Chirachanchai S., J. Am. Chem. Soc., 130 (2008) 15460.

[15] Liu Y., Antaya H. and Pellerin C., J. Polym. Sci. Pt. B-Polym. Phys., 46 (2008) 1903.

[16] Nasir M. and Kataki M., Int. J. Nanosci., 8 (2009) 1222.

[17] Katta P., Alessandro M., Ramsier R. D. and Chase G. G., Nano Lett., 4 (2004) 2215.

[18] Bayan C., Levitt J. M., Miller E., Kaplan D. and Georgakoudi I., J. Appl. Phys., 105 (2009) 102042.

[19] Thompson C. J., Chase G. G., Yarin A. L. and Reneker D. H., Polymer, 48 (2007) 6913.

[20] Brasselet S. and Zyss J., C. R. Phys., 8 (2007) 165.

[21] Halbout J. M., Blit S., Donaldson W. and Tang C. L., IEEE J. Quantum Electron., QE-15 (1979) 1176. 